\title{
Intervención psicológica en la comunidad en hombres condenados por violencia de género
}

\author{
Santiago Boira ${ }^{1 *}$, Yolanda López del Hoyo ${ }^{1}$, Lucía Tomás-Aragonés ${ }^{1}$ y Ana R. Gaspar ${ }^{2}$ \\ ${ }^{1}$ Universidad de Zaragoza, España \\ 2 Ayuntamiento de Zaragoza, España
}

\begin{abstract}
Resumen: En este trabajo se presentan los resultados obtenidos en un programa de intervención psicológica dirigido a hombres condenados por violencia contra la pareja. La muestra se compone de 62 hombres que se distribuyen en tres condiciones de tratamiento: Terapia Grupal Abierta $(N$ =15); Terapia Grupal Estructurada $(N=16)$; Terapia Individual $(N=16)$ y un grupo de control $(N=15)$. La evaluación del efecto del programa se lleva a cabo mediante un diseño cuasi experimental y medidas antes y después de la intervención. En relación con las variables analizadas, los resultados no son concluyentes, si bien se identifican diferencias entre las condiciones de tratamiento. Los resultados muestran diferencias pre y posttratamiento en la valoración de los logros de los participantes entre los formatos individual y grupal. En el formato grupal, la condición de Terapia Grupal Estructurada muestra valores de cambio más significativos en los cuestionarios administrados. Por otra parte, en el post-tratamiento, aumentan en la condición de Terapia Individual las medias en las siguientes variables del SCL-90-R (Sensibilidad interpersonal, Depresión, Hostilidad, IGS PSDI y TP). Se discute la pertinencia del uso de cuestionarios de autoinforme en la evaluación de este tipo de programas.

Palabras clave: Violencia contra la pareja; hombres agresores; programas de intervención por mandato judicial; cuasi-experimento.
\end{abstract}

\section{Introducción}

La experiencia en la aplicación y la valoración de programas de intervención psicológica dirigidos a hombres condenados por violencia contra la pareja en España es reciente. En el ámbito internacional se han realizado diferentes revisiones sistemáticas de este tipo de programas desarrollados tanto en el medio comunitario como dentro de la prisión (Aldarondo, 2002; Babcock, Green y Robie, 2004; Feder y Wilson, 2005; Gondolf, 2004; Lemire, Rondeau, Brochu, Schneeberger y Brodeur, 1996; Murphy y Ting, 2010; Sartin, Hansen y Huss, 2006; Saunders, 2008). Respecto a su efectividad, dichas revisiones no han proporcionado resultados concluyentes.

Uno de los primeros estudios sistemáticos sobre la eficacia de los programas de intervención con agresores fue llevado a cabo por Lemire et al. (1996). A partir de la revisión de las principales bases de datos internacionales, dichos autores analizan 126 artículos referidos a la eficacia de estos programas. Los resultados que obtienen son positivos respecto a su efecto en la reducción de la reincidencia. En las conclusiones se insiste en la importancia de valorar aspectos como la voluntariedad u obligatoriedad en la realización del programa, la motivación del agresor o el papel que la familia puede desempeñar en el proceso de rehabilitación.

* Dirección para correspondencia [Correspondence address]: Santiago Boira. Facultad de Ciencias Sociales y del Trabajo. Universidad de Zaragoza. C/ Violante de Hungría, 23. 50009-Zaragoza (España). E-mail: sboira@unizar.es
Title: Efficacy of different treatment modalities in men convicted of intimate partner violence.

Abstract: The results obtained in a psychological intervention program for men convicted of intimate partner violence are presented in this study. The sample consists of 62 men distributed into three treatment modalities: unstructured group therapy $(N=15)$; structured group therapy $(N=16)$; individual therapy $(N=16)$, and control group $(N=15)$. A quasiexperimental design with pre and post intervention assessment is used to measure the effect of the treatment modality. Although the results obtained are not conclusive in the variables analysed, some differences between treatment options are observed. Pre and post differences are observed between individual and group therapy regarding participants' goal achievements. When comparing the two group formats, the structured therapy group shows more significant values of change in the administered questionnaires. In the individual therapy format, post treatment measures show higher values in the following scores of the SCL-90-R (Interpersonal Sensitivity, Depression, Hostility, GSI, PSDI and PST). We discuss the relevance of using self-report questionnaires in evaluating such programs.

Key words: Intimate partner violence; male batterers; court-mandated intervention programs; quasi-experiment.

Por otra parte, otro importante trabajo de revisión fue realizado por Babcock et al. (2004). Se examinaron 22 programas evaluados a partir de un diseño experimental o cuasiexperimental. El análisis incluyó programas basados en modelos psicoeducativos de género y programas de orientación cognitivo-conductual. Ni la orientación ni el tipo de diseño produjeron diferencias estadísticamente significativas en los resultados. En esta misma línea concluyó el meta-análisis realizado por Feder y Wilson (2005) después de analizar 10 programas aplicados en la comunidad por mandato judicial. Mientras que la eficacia del programa fue mínima en las investigaciones apoyadas en la información proporcionada por la víctima, los resultados basados en los abandonos del programa fueron más positivos.

No obstante, las principales revisiones realizadas han detectado deficiencias en los diseños de evaluación que pueden distorsionar los resultados. Aspectos como la asignación de los sujetos a los grupos o el modo en que cada programa define el alta terapéutica pueden interferir decididamente en el proceso (Gondolf, 2004). En relación a la falta de rigor, Saunders (2008) planteó conclusiones similares después de revisar más de 35 programas de tratamiento.

En España son todavía recientes los estudios que evalúan tanto la trayectoria de algunos programas de tratamiento (e.g., Arce y Fariña, 2006; Boira, 2010; Expósito y Ruiz, 2009; Lila et al., 2010; Romero, Rodríguez y Echauri, 2005) como las variables relacionadas con el abandono y la eficacia de la intervención (e.g., Boira y Jodrá, 2010; Echeburúa y Fernández-Montalvo, 2009; Echeburúa, Sarasua, Zubizarreta, Amor y Corral, 2010; Echeburúa, Sarasua, Zubizarreta y Corral, 2009; Fernández-Montalvo, López-Goñi y Arteaga, 
2011; Loinaz, Irureta y Domenech, 2011; Loinaz, Torrubia, Echeburúa, Navarro y Fernández, 2009; Pérez y Martínez, 2010).

Entre ellos, Echeburúa y Fernández-Montalvo (2009) realizaron un estudio cuasi-experimental con una muestra de 148 hombres que cumplían condena en 18 cárceles españolas. Según los resultados presentados, una vez concluido el programa se produjo una modificación significativa de los sesgos cognitivos sobre la mujer y el uso de la violencia, disminuyó la sintomatología psicopatológica, la impulsividad y la ira de los sujetos en tratamiento, y aumentó significativamente la autoestima de los participantes. Asimismo, Echeburúa et al. (2009) y Echeburúa et al. (2010) han evaluado, a partir de un diseño experimental de medidas repetidas, un programa de orientación cognitivo-conductual y aplicado en formato individual en el que los hombres accedieron voluntariamente. En este caso, se valoraron las variables predictoras del rechazo, abandono y fracaso terapéutico y se analizaron las recaídas en función del número de hombres que completaron con éxito el tratamiento. Se produjo un nivel alto de rechazos y abandonos. Sin embargo, en aquéllos que completaron el programa se redujo significativamente el número de recaídas. Boira y Jodrá (2010) realizaron un estudio ex post facto a partir de una muestra de 118 hombres que participaron voluntariamente y analizaron las posibles relaciones de dependencia o de independencia entre algunas de las características de los hombres y el abandono del tratamiento. Los resultados mostraron una relación de dependencia entre la posibilidad de abandono y la existencia de historia psicológica o psiquiátrica, la pauta de consumo de alcohol y el consumo de otras drogas.

Respecto a la reincidencia, Pérez y Martínez (2010) analizaron una muestra de 170 hombres condenados por un delito de violencia de género que participaron en un programa formativo en la comunidad como consecuencia de una ejecutoria penal. La tasa de reincidencia encontrada fue baja $(8.8 \%)$. Por otra parte, los resultados mostraron que la posibilidad de reincidencia estaba asociada con las siguientes variables: alcoholismo, resistencia al cambio, haber sido testigo de malos tratos en la infancia, período de desempleo sin justificar y consumo de drogas durante la comisión del delito. En esta misma línea, Loinaz et al. (2011) realizaron un seguimiento de un grupo de 40 hombres condenados a una pena de prisión una vez que salieron de la cárcel. En este caso, la reincidencia en la comisión de algún nuevo delito relacionado con la violencia contra la pareja fue del 15\%. Dichos autores señalan además la existencia de diferentes tasas de reincidencia en función de las características del agresor.

En relación con la eficacia de los tratamientos, en trabajos recientes se insiste en considerar los diferentes tipos de agresores (Amor, Echeburúa y Loinaz, 2009; Bender y Roberts, 2007; Huss y Ralston, 2008; Loinaz, Echeburúa y Torrubia, 2010), la diversidad de programas y de contextos en los que se desarrolla la intervención (Saunders, 2008) y la importancia de mantener al agresor en el programa abordando los aspectos relacionados con su adherencia y motivación hacia el tratamiento (Alexander y Morris, 2008; Eckhardt, Babcock y Homack, 2004; Murphy y Ting, 2010; Olver, Stockdale y Wormith, 2011). Aunque el grupo es el formato habitual de aplicación de estos programas, Murphy y Meis (2008) plantean la necesidad de considerar el formato individual de intervención y sugieren algunas ventajas respecto a la intervención grupal que pueden mejorar el vínculo terapéutico y la predisposición hacia el cambio del agresor.

En este contexto de evaluación se identifican variables cada vez más específicas y, como consecuencia, algunos aspectos como la orientación teórica, el modo de aplicación del programa, el medio (comunitario o penitenciario) o el formato de intervención (individual o grupal), pueden desempeñar un papel esencial en la mejora de los resultados. El objetivo del presente trabajo es valorar la aplicación de un programa de tratamiento en la comunidad para hombres condenados por un delito de violencia contra la pareja, en el que los participantes deben someterse obligatoriamente a la intervención como forma de evitar la ejecución de una pena privativa de libertad. Además de evaluar la eficacia del programa y la reincidencia de los agresores durante los 18 meses posteriores a la finalización del programa, se valora la influencia que el formato de tratamiento aplicado puede tener en los resultados obtenidos. Para ello, se han considerado tres condiciones de tratamiento en las que se ha valorado el formato individual o grupal y el modo, abierto o estructurado, en que el programa ha sido aplicado. Se ha empleado un diseño cuasi-experimental de evaluación pre-post con cuatro grupos, uno de cuasi-control (Montero y León, 2007).

\section{Método}

\section{Participantes}

La muestra se compuso de 62 hombres que participaron en un programa de intervención psicológica aplicado en el año 2008 en el Servicio de Gestión de Penas y Medidas Alternativas de Zaragoza (España). Los criterios de inclusión fueron los siguientes: a) hombres condenados por un delito de violencia de género a los que se les ha suspendido o sustituido la ejecución de la pena de prisión e impuesto como medida obligatoria la participación en el programa; y b) consentimiento informado para participar en el estudio. Igualmente, se consideraron los siguientes criterios de exclusión: a) presencia de un trastorno médico o psicológico grave, problemas idiomáticos, intelectuales o de abuso de sustancias que pudieran impedir el correcto desarrollo del protocolo de evaluación y del programa; b) imposibilidad para acudir a las sesiones de intervención; y c) negativa a participar.

Las principales características sociodemográficas se muestran en la Tabla 1. 
$\underline{\text { Tabla 1. Variables sociodemográficas. }}$

\begin{tabular}{|c|c|c|c|c|c|c|}
\hline & & $\begin{array}{c}\text { Terapia Individual } \\
\quad N=16\end{array}$ & $\begin{array}{r}\text { Terapia Grupal } \\
\text { Abierta. } N=15\end{array}$ & $\begin{array}{c}\text { Terapia Grupal } \\
\text { Estructurada } N=16\end{array}$ & $\begin{array}{c}\text { Grupo de Control } \\
N=15\end{array}$ & $\begin{array}{c}\text { Estadístico } \\
\text { (significación) }\end{array}$ \\
\hline$\overline{\text { Edad }}$ & Media $(D T)$ & $39.4(8.6)$ & $44.3(13.6)$ & $34.9(6.8)$ & $40.2(9.9)$ & $2.32(.085)$ \\
\hline \multirow[t]{5}{*}{ Procedencia } & $N(\%)$ & & & & & \multirow{5}{*}{$9.70(.376)$} \\
\hline & España & $8(50.0)$ & $13(86.7)$ & $7(43.8)$ & $9(60.0)$ & \\
\hline & U.E. & $2(12.5)$ & $0(0)$ & $2(12.5)$ & $1(6.7)$ & \\
\hline & Magreb & $1(6.3)$ & $0(0)$ & $2(12.5)$ & $0(0)$ & \\
\hline & América & $5(31.3)$ & $2(13.3)$ & $5(31.3)$ & $5(33.3)$ & \\
\hline \multirow[t]{6}{*}{ Estudios } & $N(\%)$ & & & & & \multirow{6}{*}{$11.9(.453)$} \\
\hline & Sin estudios & $0(0)$ & $1(7.1)$ & $0(0)$ & $0(0)$ & \\
\hline & Cert. Escolar & $2(12.5)$ & $1(7.1)$ & $3(18.8)$ & $0(0)$ & \\
\hline & EGB/ESO/FP I & $6(37.5)$ & $9(64.3)$ & $7(43.8)$ & $7(46.7)$ & \\
\hline & Bach./FP II & $8(50.0)$ & $2(14.3)$ & $5(31.3)$ & $7(46.7)$ & \\
\hline & $\begin{array}{l}\text { Tít. Univ. } \\
\qquad N(\%)\end{array}$ & $0(0)$ & $1(7.1)$ & $1(6.3)$ & $1(6.7)$ & \\
\hline \multirow{4}{*}{ Convivencia } & Solo & $8(50.0)$ & $4(26.7)$ & $9(56.3)$ & $5(35.7)$ & \multirow{4}{*}{$6.07(.733)$} \\
\hline & Víctima & $2(12.5)$ & $1(6.7)$ & $1(6.3)$ & $1(7.1)$ & \\
\hline & Nueva pareja & $3(18.8)$ & $4(26.7)$ & $1(6.3)$ & $4(28.6)$ & \\
\hline & Familiar & $3(18.8)$ & $6(40.0)$ & $5(31.3)$ & $4(28.6)$ & \\
\hline \multicolumn{7}{|c|}{ Tratamiento psicológico previo $N(\%)$} \\
\hline & Sí & $2(12.5)$ & $0(0)$ & $1(6.7)$ & $3(20.0)$ & \multirow{2}{*}{$3.19(.363)$} \\
\hline & No & $14(87.5)$ & $12(100.0)$ & $14(93.3)$ & $12(80.0)$ & \\
\hline \multicolumn{7}{|c|}{ Tiene contacto con la víctima $N(\%)$} \\
\hline & No & $12(80.0)$ & $9(64.3)$ & $7(46.7)$ & $6(60.0)$ & \multirow{2}{*}{$3.62(.305)$} \\
\hline & Sí & $3(20.0)$ & $5(35.7)$ & $8(53.3)$ & $4(40.0)$ & \\
\hline
\end{tabular}

Para minimizar los posibles sesgos que se pudieran dar en la composición de los grupos experimentales, se han analizado las posibles diferencias observadas en las variables sociodemográficas. Para la variable edad se ha empleado la técnica del Análisis de Varianza y para el resto la prueba Chicuadrado. En relación con las variables consideradas no existen diferencias estadísticamente significativas entre los grupos de tratamiento y el grupo de control.

Tanto los participantes en las condiciones de tratamiento como en el grupo de control se encontraban a la espera de realizar el programa. La lista de espera se forma a partir de las ejecutorias que llegan del juzgado al Servicio de Gestión de Penas y Medidas Alternativas. En función del orden de llegada, los sujetos se asignaron a los grupos de tratamiento. El grupo control se formó a partir de los siguientes penados de la lista pendientes de realizar el programa. Dichos penados realizaron el tratamiento con posterioridad a su participación en este estudio.

\section{Instrumentos}

Los instrumentos seleccionados para la medida de la eficacia de la intervención valoran algunos aspectos que se han considerado esenciales en este tipo de intervención como son la expectativa sobre el tratamiento y la predisposición al cambio, variables psicopatológicas o los aspectos del funcionamiento psicológico relacionados con la dinámica violenta.
Expectativa y satisfacción sobre el tratamiento y disponibilidad al cambio

- Cuestionario de expectativas ante el tratamiento. Se trata de una escala de 4 ítems con 7 alternativas de respuesta en una escala de tipo Likert desde 0 (nada) a 7 (mucho). El objetivo es obtener información sobre las expectativas del sujeto antes de comenzar el programa (forma pretest) y su satisfacción a la finalización (forma postest) respecto a algunos aspectos significativos de la intervención: a) obtención de ayuda en el tratamiento para resolver los problemas, b) mejora en la expresión de las ideas y los sentimientos, c) aprendizaje en la resolución de conflictos; y d) mejora en la manera de discrepar y de respetar el punto de vista de los otros. Se considera que la mejoría en estas áreas puede ser importante en la prevención de la violencia (Dutton y Strachan, 1987; Maiuro, Cahn y Vitaliano, 1986). - Escala de Evaluación para el Cambio de la Universidad Rhode Island (URICA) (McConnaughy, DiClemente, Prochaska y Velicer, 1989; versión española de Rojas y Espinoza, 2008). Este cuestionario se basa en el modelo teórico desarrollado por Prochaska y DiClemente (1982) y valora la posición motivacional o actitud hacia el cambio de una determinada conducta problema. Se trata de una escala de 32 ítems en donde los sujetos muestran su grado de acuerdo sobre una serie de frases en una escala de tipo likert de 5 puntos $(1=$ completamente en desacuerdo $5=$ completamente de acuerdo). Se valoran cuatro momentos en la disposición hacia el cambio: precontemplación, contemplación, actuación y mantenimiento. Los coeficientes de confiabilidad de las cuatro subescalas del URICA determinados mediante el 
método de Consistencia Interna de Kuder Richardson son adecuados y oscilan entre .83 y .97 .

\section{Alteraciones psicopatológicas}

- Listado de Síntomas (SCL-90-R) (Derogatis, 1975; versión española de González de Rivera, 2002). Se trata de un cuestionario autoaplicado para la detección y medida de síntomas psicopatológicos. Se compone de 90 ítems puntuados sobre una escala de tipo Likert con 5 opciones de respuesta en función de la intensidad del síntoma desde 0 (nada) a 4 (extremo). Se evalúan nueve dimensiones primarias: somatización, obsesión-compulsión, sensitividad interpersonal, depresión, ansiedad, hostilidad, ansiedad fóbica, ideación paranoide y psicoticismo. Además, se aportan tres índices globales: indice de gravedad global, indice de malestar positivo y total de sintomas positivos. Los coeficientes de consistencia interna y de fiabilidad test-retest se sitúan entre .81 y .90 y el coeficiente de estabilidad temporal oscila entre .78 y .90 . Este cuestionario ha sido utilizado en estudios anteriores en el entorno de evaluación de los agresores de pareja (Echeburúa y Fernández-Montalvo, 2009).

\section{Variables de personalidady distorsiones cognitivas}

- Inventario de Hostilidad (BDHI) (Buss y Durkee, 1957; versión española de Montalvan et al., 2001). Este cuestionario consta de 75 ítems de respuesta dicotómica (verdadero o falso). Cada respuesta valorada como verdadera equivale a 1 punto y cada respuesta valorada como falsa a 0 puntos. Los ítems se agrupan en siete subescalas que evalúan diferentes aspectos relacionados con la hostilidad: propensión al ataque o asalto, hostilidad verbal, hostilidad indirecta, irritabilidad, negativismo, resentimiento, sospecha y sinceridad. El coeficiente de fiabilidad test-retest de este instrumento es de .92.

- Índice de Reactividad Interpersonal (IRI) (Davis, 1980; versión española de Pérez-Albéniz, De Paúl, Etxeberría, Montes y Torres, 2003). Este cuestionario está compuesto por 28 ítems valorados en una escala tipo Likert de 5 puntos desde $1=$ no me describe bien a $5=$ me describe muy bien $\mathrm{y}$ distribuidos en cuatro subescalas que miden distintas dimensiones del concepto global de empatía: toma de perspecti- va, fantasía, preocupación empática y malestar personal. Los coeficientes de consistencia interna de la versión española obtenidos mediante el coeficiente alfa $(\alpha)$ de Cronbach son inferiores a los logrados por Davis (1980) y oscilan entre .63 y .80 . Esta escala ha sido utilizada con anterioridad en estudios con agresores siendo sensible al cambio terapéutico (Echeburúa y Fernández-Montalvo, 2009; Echeburúa, Fernández-Montalvo y Corral, 2008; Loinaz, 2010).

- Inventario de Pensamientos Distorsionados sobre la Mujer y el Uso de la Violencia (Echeburúa y Corral, 1998). Se trata de un listado de 29 ítems de respuesta dicotómica (sí o no) que trata de detectar los pensamientos irracionales del hombre. De estos ítems, 13 se refieren a pensamientos distorsionados sobre la mujer y 16 al uso de la violencia como forma aceptable de solucionar conflictos. Este inventario ha sido utilizado con muestras españolas en diferentes estudios con agresores en el ámbito de pareja (Echeburúa y Fernández-Montalvo, 2009; Echeburúa, FernándezMontalvo y Amor, 2006; Loinaz, 2010). Para este trabajo se ha considerado el número de pensamientos distorsionados presentes en cada individuo.

\section{Tratamiento}

El programa aplicado, de orientación cognitivoconductual, es una adaptación del desarrollado por la Secretaría General de Instituciones Penitenciarias para su ejecución dentro de la prisión (Dirección General de Instituciones Penitenciarias, 2005).

Para la aplicación del tratamiento se diseñaron tres grupos, dos en formato grupal (Terapia Grupal Abierta y Terapia Grupal Estructurada) y uno en formato individual (Terapia Individual). En la condición de Terapia Grupal Estructurada las áreas de trabajo se abordaron siguiendo un orden estructurado en función de la secuencia marcada en el programa. En la condición de Terapia Grupal Abierta los terapeutas decidieron el área de trabajo de cada sesión en función de la evolución del grupo.

Los bloques temáticos del programa y las áreas de trabajo psicológico con las sesiones empleadas en cada condición grupal se muestran en la Tabla 2.

Tabla 2. Programa de tratamiento. Unidades y áreas de intervención (DGIIPP, 2005).

Unidades temáticas
Bloque 1. Asunción de la responsabilidad

Bloque 2. Control de emociones

Bloque 3. Entrenamiento en habilidades

Bloque 4. Cierre
Areas de trabajo $\left(\mathrm{N}^{\circ}\right.$ de sesiones)

Asunción de la responsabilidad y mecanismos de defensa (2)

Identificación y expresión de emociones (2)

Empatía con la víctima (2)

Distorsiones cognitivas y creencias irracionales (2)

Ansiedad (1)

Celos (1)

Ira y resentimiento (2)

Habilidades de relación y comunicación (2)

Solución de problemas (2)

Educación sexual (1)

Estilo de vida positivo (1)

Prevención de recaídas (2) 
El programa se desarrolló durante 24 sesiones: 2 de evaluación, 20 de tratamiento y 2 de seguimiento a los 3 y 6 meses. La frecuencia de las sesiones fue semanal con una duración de 2 horas para los grupos y de aproximadamente 1 hora para la condición individual.

\section{Procedimiento}

El programa lo aplicaron psicólogos con experiencia clínica y especializados en violencia dentro de la pareja. La distribución de los participantes entre las condiciones de tratamiento no fue aleatoria, se efectuó considerando sus horarios laborales. El grupo de Terapia Grupal Abierta se realizó en horario de mañana y el grupo de Terapia Grupal Estructurada por la tarde. En la condición individual, terapeuta y participante acordaron el horario de las entrevistas.

Los datos pretratamiento se obtuvieron durante las dos sesiones iniciales de evaluación y los datos postratamiento en la última sesión de tratamiento. En las entrevistas de seguimiento se preguntó a los hombres si había existido contacto con la víctima y se valoró la existencia de nuevos incidentes de violencia a partir de la información ofrecida por el penado. Asimismo, se consultó la base de datos del Sistema de Seguimiento Integral en los casos de Violencia de Género (Sistema VdG), gestionada por el Gabinete de Estudios de Seguridad Interior del Ministerio del Interior, para comprobar si los participantes habían sido nuevamente detenidos por un delito de Violencia de Género durante los 18 meses siguientes a la finalización del programa.

Posteriormente, los datos se codificaron mediante un procedimiento de encriptación para asegurar la confidencialidad de los participantes. El análisis estadístico comenzó por una exploración descriptiva de las variables seleccionadas para este trabajo. Mediante la prueba de KolmogorovSmirnov/Shapiro-Wilks se comprobó el ajuste a la distribución normal de los valores de las diferentes escalas analizadas. Se optó por un estudio no paramétrico de los datos de- bido a que el ajuste a la distribución normal en todas las pruebas aplicadas resultó inadecuado salvo en una de las cuatro subescalas del cuestionario de Evaluación para el Cambio (URICA). Como consecuencia, se realizó un análisis multivariante mediante la prueba de Wilcoxon para dos muestras relacionadas para determinar la influencia del programa de tratamiento sobre las variables estudiadas. Además, se examinó en las diferentes escalas la presencia de puntuaciones atípicas que hubieran podido distorsionar los resultados obtenidos. Sin embargo, y pese a la presencia de alguna puntuación atípica, éstas no influyeron de forma determinante. En la sección dedicada a la presentación de los resultados se han reflejado los p-valores menores de .1 y mayores de .05 puesto que pueden sugerir algunas tendencias de interés para futuras investigaciones.

\section{Resultados}

\section{Abandonos del programa, relación con la víctima y nuevas detenciones}

De los 47 participantes en el programa 43 lo completaron $(91.49 \%)$. Respecto a la relación mantenida con la víctima una vez finalizado el programa, el $65.12 \%(N=30)$ manifestó no haber tenido ningún tipo de contacto. De los participantes que sí lo admitieron, ninguno declaró que hubiera sido conflictivo. Finalmente, según los datos del Sistema $\mathrm{VdG}$, en el $93.62 \%(N=44)$ de los participantes en el programa no constaban nuevas diligencias policiales ni denuncias por motivos de violencia de género en los 18 meses posteriores al tratamiento.

\section{Expectativa y predisposición al cambio}

Los resultados que valoran la expectativa en el tratamiento y la predisposición al cambio (URICA) se muestran en la Tabla 3.

Tabla 3. Expectativa sobre el tratamiento y predisposición al cambio.

\begin{tabular}{|c|c|c|c|c|c|c|c|c|c|c|c|c|}
\hline & \multicolumn{3}{|c|}{$\begin{array}{l}\text { Terapia Individual } \\
N=16\end{array}$} & \multicolumn{3}{|c|}{$\begin{array}{l}\text { Terapia Grupal } \\
\text { Abierta. } N=15\end{array}$} & \multicolumn{3}{|c|}{$\begin{array}{l}\text { Terapia Grupal } \\
\text { Estructurada. } N=16\end{array}$} & \multicolumn{3}{|c|}{$\begin{array}{l}\text { Grupo de Control } \\
N=15\end{array}$} \\
\hline $\begin{array}{l}\text { Expectativa sobre } \\
\text { el tratamiento }\end{array}$ & $\begin{array}{l}\text { Pre. } \\
M(D T)\end{array}$ & $\begin{array}{l}\text { Post. } \\
M(D T)\end{array}$ & $Z$ & $\begin{array}{l}\text { Pre. } \\
M(D T)\end{array}$ & $\begin{array}{l}\text { Post. } \\
M(D T)\end{array}$ & $Z$ & $\begin{array}{l}\text { Pre. } \\
M(D T)\end{array}$ & $\begin{array}{l}\text { Post. } \\
M(D T)\end{array}$ & $Z$ & $\begin{array}{l}\text { Pre. } \\
M(D T)\end{array}$ & $\begin{array}{l}\text { Post. } \\
M(D T)\end{array}$ & $Z$ \\
\hline $\begin{array}{l}\text { Resolver el pro- } \\
\text { blema }\end{array}$ & $3.7(3.0)$ & $4.5(2.2)$ & -1.13 & $4.5(2.2)$ & $4.2(2.3)$ & -.11 & $4.9(2.3)$ & $4.3(1.7)$ & -1.41 & & & \\
\hline $\begin{array}{l}\text { Ideas y sentimien } \\
\text { tos }\end{array}$ & $2.8(2.5)$ & $5.0(2.1)$ & $-2.78^{* *}$ & $4.2(2.3)$ & $4.0(2.4)$ & -.21 & $4.5(2.1)$ & $4.2(1.7)$ & -.59 & & & \\
\hline $\begin{array}{l}\text { Resolver los con } \\
\text { flictos }\end{array}$ & $3.3(2.8)$ & $5.0(2.3)$ & $-2.61 * *$ & $4.6(2.6)$ & $4.6(2.3)$ & -.84 & $4.6(2.3)$ & $5.0(2.0)$ & -1.13 & & & \\
\hline Forma de discrepar & $3.8(2.9)$ & $5.1(2.0)$ & $-2.15^{*}$ & $4.5(2.5)$ & $5.1(2.3)$ & -1.58 & $4.6(2.1)$ & $4.8(2.1)$ & -.46 & & & \\
\hline URICA & & & & & & & & & & & & \\
\hline Precontemplación & $58.8(17.4)$ & $59.6(12.9)$ & -.54 & $57.0(18.1)$ & $64.7(15.8)$ & -1.61 & $50.3(10.8)$ & $56.1(9.1)$ & $-2.20 *$ & * 57.8 (11) & $53.8(16.2)$ & -1.12 \\
\hline Contemplación & $64.6(24.3)$ & $71.4(21.7)$ & -1.45 & $63.5(23.7)$ & $59.1(20.6)$ & -.11 & $70.0(17.8)$ & $69.8(16.1)$ & -.12 & $72.5(19.6)$ & $70.6(17.6)$ & -.31 \\
\hline Actuación & $53.6(26.8)$ & $62.9(21.7)$ & -1.28 & $59.0(27.7)$ & $54.8(22.3)$ & -.13 & $61.2(25.3)$ & $62.8(19.8)$ & -.35 & $63.1(21.8)$ & $56.5(26.2)$ & -1.39 \\
\hline Mantenimiento & $37.3(15.6)$ & $45.6(17.5)$ & $-1.85 \sim$ & $51.8(24.6)$ & $42.4(17.8)$ & -.58 & $40.4(18.9)$ & $51.8(13.3)$ & $-2.07 *$ & $43.5(15.4)$ & $42.3(19.1)$ & -.14 \\
\hline
\end{tabular}


En relación con la diferencia entre la expectativa en el tratamiento de los participantes y su satisfacción posterior se obtuvieron diferencias estadísticamente significativas en la condición de tratamiento individual, en la que la media aumentó en 3 de las 4 variables estudiadas: expresión de ideas y sentimientos $(p<.01)$, aprendizaje de resolución de conflictos $(p<$ $.01)$ y mejora en la forma de discrepar $(p<.05)$. Por otra parte, en los resultados obtenidos en el cuestionario URICA aumentaron las diferencias en el estadio de mantenimiento del cambio en la condición de tratamiento individual $(p<.1)$.y en la condición de Terapia Grupal Estructurada $(p<.05)$. Asimismo, se produjo un aumento de la media en el estadio de precontemplación en la condición de Terapia Grupal Estructurada ( $p$ $<.05)$.

\section{Síntomas de psicopatología}

En el SCL-90-R, los resultados más significativos se produjeron en la condición de Terapia Grupal Estructurada. Se observó una disminución de la media en las escalas: depresión $(p<.01)$, indice global de severidad (IGS) $(p<.05)$ e indice de total de sintomas positivos (TP) $(p<.05)$. También aumentó la media de la escala de hostilidad en la condición de tratamiento individual $(p<.1)$ y disminuyó en las escalas de sensibilidad interpersonal e ideación paranoide del grupo de control $(p<.1)$ (véase la Tabla 4).

$\underline{\text { Tabla 4. Síntomas de psicopatología. }}$

\begin{tabular}{|c|c|c|c|c|c|c|c|c|c|c|c|c|}
\hline \multirow[b]{2}{*}{ SCL-90-R } & \multicolumn{3}{|c|}{$\begin{array}{l}\text { Terapia Individual } \\
N=16\end{array}$} & \multicolumn{3}{|c|}{$\begin{array}{l}\text { Terapia Grupal } \\
\text { Abierta. } N=15\end{array}$} & \multicolumn{3}{|c|}{$\begin{array}{l}\text { Terapia Grupal } \\
\text { Estructurada. } N=16\end{array}$} & \multicolumn{3}{|c|}{$\begin{array}{l}\text { Grupo de Control } \\
N=15\end{array}$} \\
\hline & $\begin{array}{l}\text { Pre. } \\
M(D T)\end{array}$ & $\begin{array}{l}\text { Post. } \\
M(D T)\end{array}$ & $Z$ & $\begin{array}{l}\text { Pre. } \\
M(D T)\end{array}$ & $\begin{array}{l}\text { Post. } \\
M(D T)\end{array}$ & $Z$ & $\begin{array}{l}\text { Pre. } \\
M(D T)\end{array}$ & $\begin{array}{l}\text { Post. } \\
M(D T)\end{array}$ & $Z$ & $\begin{array}{l}\text { Pre. } \\
M(D T)\end{array}$ & $\begin{array}{l}\text { Post. } \\
M(D T)\end{array}$ & $Z$ \\
\hline & $39.0(25.4)$ & $32.3(25.9)$ & -.56 & $41.3(30.3)$ & $33.4(31.1)$ & -.49 & $40.9(25.8)$ & $31.3(18.4)$ & -1.05 & $40.6(28.3)$ & $38.2(30.5)$ & -.54 \\
\hline $\begin{array}{l}\text { Obsesión- compul- } \\
\text { sión }\end{array}$ & 39 & 3 & 1 & 3 & 3 & 2 & 35.9 (24.6) & 2 & -1.29 & 3 & 3 & -.66 \\
\hline $\begin{array}{l}\text { ensibilidad inter- } \\
\text { ersonal }\end{array}$ & 3 & $42.0(21.0)$ & -.98 & $37.6(21.5)$ & $29.6(18.9)$ & 31 & $29.1(20.0)$ & $29.6(22.2)$ & -.31 & $44.3(19.8)$ & $35.0(21.7)$ & $-1.74 \sim$ \\
\hline epresión & 38.6 & & 0 & & 2 & -.87 & 47.8 & & $-3.17 * *$ & & & -1.42 \\
\hline & 35. & 34.6 & -.51 & 33. & 20.3 & -1.25 & 32. & 20. & -1.10 & & 27. & -.07 \\
\hline & 21. & 25.6 & $-1.35 \sim$ & 31. & 17.6 & -1.73 & 23.1 & 23. & -.18 & $5.8)$ & 17. & -1.43 \\
\hline ica & 27.3 & 26.6 & -.24 & 26. & 20 & -.84 & 19.4 & 23 & -.11 & $0.9)$ & 35. & -1.07 \\
\hline ide & 49.3 & 48.9 & -1.17 & 35 & 33 & -.42 & 50.3 & 39. & -1.23 & 50.1 & 43.8 & -1.84 \\
\hline Psicotici & & & & & & -.42 & 39. & & -1 & 7.1) & 38.5 & -.35 \\
\hline IGS & & & -.4 & & & -1.14 & 32.5 & 20 & $-2.27 *$ & $3.8)$ & 30.4 & -.98 \\
\hline TP & & $40.0(22$. & -1.22 & $35.6(29.9)$ & $35.0(30$ & 0 & $36.2(24.6)$ & $29.3(26.7)$ & $-2.08^{*}$ & $46.3(24.4)$ & $38.8(27.3)$ & -1.24 \\
\hline PSDI & $27.6(22.5)$ & $28.0(20.5)$ & -.27 & $40.6(28.4)$ & $19.6(16.6)$ & -1.48 & $22.2(15.7)$ & $17.0(15.3)$ & -1.55 & $26.3(14.9)$ & $26.4(26.7)$ & -.56 \\
\hline
\end{tabular}

Nota. IGS: Índice Global de Severidad; PSDI: Índice Positivo de Malestar; TP: Total de Síntomas Positivos; Z: transformación de la puntuación t de la prueba de Wilcoxon en muestras grandes $(>25)$.

$\sim p<.1 ; * p<.05 ; * * p<.01$.

\section{Variables de personalidad y distorsiones cognitivas}

Los resultados sobre las variables de personalidad y la presencia de distorsiones cognitivas sobre la mujer y el uso de la violencia los resultados se presentan en la Tabla 5.

Tabla 5. Variables de personalidad y distorsiones cognitivas.

\begin{tabular}{|c|c|c|c|c|c|c|c|c|c|c|c|c|}
\hline \multirow[b]{2}{*}{ IRI } & \multicolumn{3}{|c|}{$\begin{array}{l}\text { Terapia Individual } \\
N=16\end{array}$} & \multicolumn{3}{|c|}{$\begin{array}{l}\text { Terapia Grupal } \\
\text { Abierta } N=15\end{array}$} & \multicolumn{3}{|c|}{$\begin{array}{l}\text { Terapia Grupal } \\
\text { Estructurada } N=16\end{array}$} & \multicolumn{3}{|c|}{$\begin{array}{l}\text { Grupo de Control } \\
N=15\end{array}$} \\
\hline & $\begin{array}{l}\text { Pre. } \\
M(D T)\end{array}$ & $\begin{array}{l}\text { Post. } \\
M(D T)\end{array}$ & $Z$ & $\begin{array}{l}\text { Pre. } \\
M(D T)\end{array}$ & $\begin{array}{l}\text { Post. } \\
M(D T)\end{array}$ & $Z$ & $\begin{array}{l}\text { Pre. } \\
M(D T)\end{array}$ & $\begin{array}{l}\text { Post. } \\
M(D T)\end{array}$ & $Z$ & $\begin{array}{l}\text { Pre. } \\
M(D T)\end{array}$ & $\begin{array}{l}\text { Post. } \\
M(D T)\end{array}$ & $Z$ \\
\hline Toma de Perspectiva & $3.3(.8)$ & $3.1(.9)$ & -.83 & $3.4(.8)$ & $3.4(.7)$ & -.97 & $3.1(.7)$ & $3.3(.6)$ & $-2.04 *$ & $3.6(.8)$ & $3.3(.6)$ & -.74 \\
\hline Fantasía & $2.2(.5)$ & $2.5(.6)$ & -1.41 & $2.6(.6)$ & $2.4(.3)$ & -1.62 & $2.1(.4)$ & $2.2(.6)$ & -.70 & $2.5(.4)$ & $2.3(.4)$ & $-1.85 \sim$ \\
\hline Empatía & $3.5(.6)$ & $3.4(.5)$ & -.44 & $3.4(.8)$ & $3.5(.3)$ & -.32 & $3.4(.6)$ & $3.4(.4)$ & -.42 & $3.6(.5)$ & $3.3(.4)$ & $-2.48^{*}$ \\
\hline Malestar personal & $1.6(.6)$ & $1.5(.7)$ & -.32 & $1.8(.6)$ & $1.8(.7)$ & -.76 & $1.7(.4)$ & $1.8(.5)$ & -.51 & $1.9(.6)$ & $1.7(.4)$ & -.47 \\
\hline
\end{tabular}

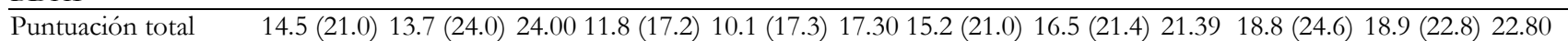
IPD

\begin{tabular}{|c|c|c|c|c|c|c|c|c|c|c|c|}
\hline Mujer & $5.4(2.1)$ & $4(2.5)$ & $-1.612 .8(1.9)$ & $3.3(1.5)$ & -.58 & $3.1(1.9)$ & $2.8(1.9)$ & -.89 & $4.7(3.1)$ & $4.8(2.2)$ & -1.34 \\
\hline Uso de la violencia & $5.8(2.7)$ & $5.2(2.8)$ & $3.7(1.7)$ & $4.7(3.7)$ & -.54 & $4.6(2.1)$ & $4.9(2.6)$ & -.27 & $5.5(2.9)$ & $5.6(1.7)$ & -.26 \\
\hline
\end{tabular}


Respecto a la medida de la empatía (IRI), se produjo un aumento de la media en la escala toma de perspectiva en la condición grupal B $(p<.05)$. Por otra parte, el grupo de control mostró una disminución de las medias en las escalas empatía $(p<.05)$ y fantasía $(p<.1)$. En relación con el cuestionario que valora la hostilidad (BDHI) no se observan diferencias significativas en ninguna de las escalas estudiadas. No obstante, se deben considerar las bajas puntuaciones medias obtenidas en todos los grupos tanto en la evaluación pretratamiento como en la postratamiento. En el Inventario de Pensamientos Distorsionados sobre la Mujer y el Uso de la Violencia tampoco se han producido cambios estadísticamente significativos en ninguna de las condiciones.

\section{Discusión}

Actualmente los programas de tratamiento en España para hombres que agreden a la pareja pueden aplicarse bajo tres condiciones: a partir de la participación voluntaria del hombre, como consecuencia de una medida judicial que sustituye o suspende una pena privativa de libertad y una vez que el hombre se encuentra en prisión. Aunque se ha publicado algún trabajo sobre la reincidencia de los agresores de pareja que han realizado un programa de tratamiento en la comunidad (Pérez y Martínez, 2010), los estudios existentes en España hasta la fecha se han centrado en programas desarrollados dentro de prisión (Echeburúa y FernándezMontalvo, 2009; Loinaz et al., 2011) o en programas en los que los hombres han participado voluntariamente (Boira y Jodrá, 2010; Echeburúa et al., 2010; Echeburúa et al., 2009). En esta investigación se evalúa la aplicación por mandato judicial de un programa de intervención psicológica en el medio comunitario dirigido a agresores condenados por un delito de violencia de género.

En líneas generales, las características sociodemográficas de la muestra son similares a las obtenidas en otras investigaciones (Boira y Jodrá, 2010; Fernández-Montalvo, Echeburúa y Amor, 2005; Redondo, Graña y González, 2009). No obstante, como se señala en otros trabajos, destaca el número creciente de hombres de procedencia no española (Fernández-Montalvo, Echauri, Martínez y Azcárate, 2011; Loinaz, 2010). Por otra parte, algunas de las pruebas aplicadas en este estudio se han utilizado en investigaciones españolas sobre agresores en la pareja. En concreto, cabe subrayar el empleo del Índice de Reactividad Interpersonal (IRI) (Echeburúa y Fernández-Montalvo, 2009; Echeburúa et al., 2008; Loinaz, 2010) y del Inventario de Pensamientos Distorsionados sobre la Mujer y el Uso de la Violencia (Echeburúa y Fernández-Montalvo, 2009; Echeburúa et al., 2006; Loinaz, 2010). Al comparar los resultados que obtuvieron en la evaluación inicial Echeburúa et al. (2009), las puntuaciones alcanzadas en el Inventario de Pensamientos Distorsionados (IPD) tampoco muestran un número elevado de distorsiones. En ambos casos, se alcanza una puntuación media más alta en la subescala de pensamientos distorsionados sobre el uso de la violencia. Sin embargo, a diferencia del estudio citado, en el trabajo que aquí se presenta las puntuaciones medias obtenidas en las dos subescalas son ligeramente superiores. Asimismo, los resultados que se obtienen en el Índice de Reactividad Interpersonal (IRI) son similares a los logrados por Loinaz (2010). En los dos estudios las puntuaciones en las subescalas de toma de perspectiva y empatía obtienen las mayores puntuaciones.

Por otra parte, el $91.49 \%$ de los hombres terminaron la intervención. En relación con estudios anteriores, el porcentaje de abandonos es menor que en los programas donde los participantes acceden voluntariamente. En ese caso, las bajas por abandono se sitúan en torno al 50\% y se producen, fundamentalmente, en las primeras sesiones (Boira, 2010; Echeburúa, y Corral, 1998; Echeburúa et al., 2009). Posiblemente sea el mandato judicial y la amenaza de ingreso en prisión lo que asegure la permanencia del hombre en la intervención. No obstante, de los hombres que terminan el programa ninguno de ellos manifiesta haber tenido algún incidente de violencia con la víctima, lo que se corresponde con el rastreo realizado de la base de datos del Sistema VdG. En este sentido, como señala Redondo (2008), los resultados de estos programas pueden valorarse no sólo por la diferencias encontradas pre y post tratamiento sino como disminución de la reincidencia. Únicamente 3 hombres (6.38\%) fueron nuevamente detenidos con posterioridad a la finalización de la intervención. Este porcentaje es bajo si se compara con la tasa general de reincidencia de este tipo de agresores (Coulter y VandeWeerd, 2009; Hilton, Harris y Rice, 2001; Olson y Stalans, 2001) y similar al obtenido por Pérez y Martínez (2010) en la valoración de la reincidencia de los agresores condenados por violencia de género después de realizar un programa en el ámbito comunitario. Sin embargo, la existencia de una denuncia o condena por parte del agresor no implica que la relación con la víctima cese (Echeburúa, Amor y Corral, 2002; Escudero, Polo, López y Aguilar, 2005; Montero, 2001). Un porcentaje significativo de los hombres $(34.88 \%)$ reconoce haber tenido algún tipo de contacto con la víctima después de finalizado el programa durante el seguimiento realizado.

En cuanto al efecto de la aplicación del programa sobre las variables estudiadas, los resultados no son concluyentes respecto a la eficacia del programa y se encuentran en la línea de las principales revisiones internacionales (Saunders, 2008). En relación con el formato de tratamiento aplicado, se producen diferencias entre la modalidad individual y las condiciones grupales. Los participantes en la intervención individual tienen una mayor percepción de los logros conseguidos en el tratamiento. Además, y frente a las condiciones grupales, se aprecia una tendencia general al aumento en las medias de las variables que muestran sintomatología psicopatológica; en alguno de estos casos este incremento es estadísticamente significativo (hostilidad en el SCL-90-R). De acuerdo con lo propuesto por Murphy y Meis (2008) la intervención individual puede facilitar el fortalecimiento de la 
alianza terapéutica, lo que puede incidir en el grado sinceridad del sujeto tanto con el terapeuta como en la posterior evaluación post-tratamiento.

Respecto a las modalidades grupales de intervención, la condición de Terapia Grupal Estructurada, en la que la presentación de los módulos de tratamiento se realizó de forma estructurada, ha mostrado valores de cambio más significativos (URICA, SCL-90-R, IRI). Sin embargo, y en relación con el cuestionario que mide aspectos específicos del comportamiento violento (BDHI), llama la atención las puntuaciones especialmente bajas obtenidas tanto en el pretest como en el postest, lo que puede indicar la poca sensibilidad de estas pruebas aplicadas en este contexto. Aunque es necesario el uso de instrumentos de evaluación cada vez más específicos (Lila, Herrero y Gracia, 2008; Meis, Murphy y Winters, 2010), debe revisarse la utilización de pruebas de autoinforme como estrategia principal no sólo para la evaluación del cambio sino también como evaluación inicial.

Sin embargo, de la interpretación de los datos obtenidos se deducen algunos temas de interés para la evaluación: a) la resistencia al tratamiento puede provocar que el sujeto falsee las pruebas; b) la necesidad de revisar la utilización de cuestionarios de autoinforme como estrategia principal en la evaluación del cambio; y c) la importancia de usar instrumentos de evaluación cada vez más específicos al contexto de intervención con este tipo de agresores. Es necesario destacar en este punto que la intervención con hombres condenados por violencia de género se caracteriza por la obligatoriedad en la realización del programa pudiendo incidir en la motivación del penado y en su adherencia al tratamiento (Ross, Polaschek y Ward, 2008; Taft, Murphy, Musser y Remington, 2004).

Los resultados presentados han puesto de manifiesto diferencias entre el formato de intervención grupal e individual. Murphy y Meis (2008) destacan que la terapia individual ha sido muy poco aplicada con hombres que agraden a la pareja y revisan las posibles ventajas frente al tratamiento grupal. Entre ellas destacan la posibilidad de mejorar la motivación, la adherencia al tratamiento de los participantes y la posibilidad de adecuar la intervención a su momento en el proceso de cambio (Levesque, Velicer, Castle y Greene, 2008). Del mismo modo, la terapia individual permite el abordaje más específico de problemas como el abuso de alcohol, los celos o los trastornos del estado de ánimo. En este sentido, algunos de los programas que en España han mane-

\section{Referencias}

Aldarondo, E. (2002). Evaluating the efficacy of interventions with men who batter. En E. Aldarondo y F. Mederos (Eds.), Programs for men who batter: Intervention and prevention strategies in a diverse society (p.p. 3-16). Kingston, New Jersey: Civic Research Institute.

Alexander, P.C. y Morris, E. (2008). Stages of change in batterers and their response to treatment. Violence and Victims, 23, 476-492.

Amor, P.J., Echeburúa, E. y Loinaz, I. (2009). ¿Se puede establecer una clasificación tipológica de los hombres violentos contra su pareja? International Journal of Clinical and Health Psychology, 9, 519-539. jado un formato individual de intervención han presentado resultados esperanzadores (Echeburúa et al., 2009).

Respecto al futuro, en el diseño de los programas será importante valorar el tipo de intervención propuesta en función de la predisposición al cambio del agresor, de sus características diferenciales y del contexto de intervención. En referencia al contexto, habrá que considerar también como un elemento relevante las actitudes y las creencias que poseen los terapeutas que realizan los programas y los diferentes agentes implicados (Cubells, Calsamiglia y Albertín, 2010; Ferrer, Bosch, Navarro, Ramis y García, 2008). De la misma forma, habrá que ampliar las estrategias de evaluación para que ofrezcan una información cualitativamente diferente y permitan una valoración más global del impacto de los tratamientos y del riesgo para la victima (Buchbinder y Eisikovits, 2008; Shamai y Buchbinder, 2010).

Para terminar, se deben señalar algunas limitaciones de este estudio. En primer lugar, se podría haber ampliado el tipo de medidas de evaluación empleadas, recogiendo la valoración de la víctima y extendiendo la evaluación de carácter cualitativo a los agresores. En investigaciones posteriores se tratará de explorar estas posibilidades. En segundo lugar, una de las principales limitaciones ha sido la asignación no aleatoria de los sujetos a los diferentes grupos quedando condicionada por los horarios laborales. En tercer lugar, en este estudio no se han considerado los posibles perfiles del agresor ni en la formación de los grupos ni en la aplicación del programa. En investigaciones recientes se apunta la necesidad de diseñar programas específicos en función de las características del grupo de participantes con el objetivo de mejorar la eficacia de la intervención (Amor et al., 2009; Bender y Roberts, 2007; Huss y Ralston, 2008; Loinaz et al., 2010). Y, por último, es necesario subrayar la dificultad para generalizar los resultados del estudio presentado, pues éstos se refieren a un grupo concreto de hombres en un contexto específico de intervención. No obstante, se considera que esta investigación puede resultar relevante para valorar el tratamiento por mandato judicial en el medio comunitario y las diferencias observadas en función del formato de tratamiento aplicado.

Agradecimientos.- Esta investigación ha sido posible gracias a un convenio de colaboración firmado entre la Secretaría General de Instituciones Penitenciarias y el Colegio Profesional de Psicólogos de Aragón (COPPA) (2008).

Arce, R. y Fariña, F. (2006). Programa Galicia de reeducación para maltratadores de género. Anuario de Psicología Jurídica, 16, 41-64.

Babcock, J.C., Green, C.E. y Robie, C. (2004). Does batterers' treatment work? A meta-analytic review of domestic violence treatment. Clinical Psychology Review, 23, 1023-1053.

Bender, K. y Roberts, A.R. (2007). Battered women versus male batterer typologies: Same or different based on evidence-based studies? Aggression and Violent Behavior, 12, 519-530.

Boira, S. (2010). Hombres maltratadores. Historias de violencia masculina. Zaragoza: Prensas Universitarias de Zaragoza. 
Boira, S. y Jodrá, P. (2010). Psicopatología, características de la violencia y abandonos en programas para hombres violentos con la pareja: resultados en un dispositivo de intervención. Psicothema, 22, 593-599.

Buchbinder, E. y Eisikovits, Z. (2008). Doing treatment: Batterers' experience of intervention. Children and Youth Services Review, 30, 616-630.

Buss, A.H. y Durkee, A. (1957). An inventory for assessing different kinds of hostility. Journal of Consulting Psychology, 21, 343-349.

Coulter, M. y VandeWeerd, C. (2009). Reducing domestic violence and other criminal recidivism: Effectiveness of a multilevel batterers intervention program. Violence and Victims, 24, 139-152.

Cubells, J., Calsamiglia, A. y Albertín, P. (2010). El ejercicio profesional en el abordaje de la violencia de género en el ámbito jurídico-penal: un análisis psicosocial. Anales de Psicología, 26, 369-377.

Davis, M.H. (1980). A multidimensional approach to individual differences in empathy. JSAS Catalog of Selected Documents in Psychology, 10, 85.

Derogatis, L.R. (1975). The SCL-90-R. Baltimore: Clinical Psychometric Research.

Dirección General de Instituciones Penitenciarias. (2005). Programa de tratamiento en prisión para agresores en el ámbito familiar. Grupo de trabajo sobre violencia de género. Madrid: Secretaría General Técnica. Ministerio del Interior.

Dutton, D.G. y Strachan, C.E. (1987). Motivational needs for power and spouse-specific assertiveness in assaultive and nonassaultive men. Violence and Victims, 2, 145-156.

Echeburúa, E. y Corral, P. (1998). Manual de violencia familiar. Madrid: S. XXI.

Echeburúa, E. y Fernández-Montalvo, J. (2009). Evaluación de un programa de tratamiento en prisión de hombres condenados por violencia grave contra la pareja. International Journal of Clinical and Health Psychology, 9, 520.

Echeburúa, E., Amor, P.J. y Corral, P. (2002). Mujeres maltratadas en convivencia prolongada con el agresor: variables relevantes. Acción Psicológica, 1,135-150.

Echeburúa, E., Fernández-Montalvo, J. y Amor, P.J. (2006). Psychological treatment of men convicted of gender violence: A pilot-study in the Spanish prisons. International Journal of Offender Therapy and Comparative Criminology, 50, 57-70.

Echeburúa, E., Fernández-Montalvo, J. y Corral, P. (2008). ¿Hay diferencias entre la violencia grave y la violencia menos grave contra la pareja?: un análisis comparativo. International Journal of Clinical and Health Psychology, $8,355-382$

Echeburúa, E., Sarasua, B., Zubizarreta, I. y Corral, P. (2009). Evaluación de la eficacia de un tratamiento cognitivo-conductual para hombres violentos contra la pareja en un marco comunitario: una experiencia de 10 años (1997-2007). International Journal of Clinical and Health Psychology, 9, 199-217.

Echeburúa, E., Sarasua, B., Zubizarreta, I., Amor, P.J. y Corral, P. (2010). Variables predictoras del rechazo, abandono y fracaso terapéutico en hombres violentos contra su pareja tratados psicológicamente en un marco comunitario. International Journal of Clinical and Health Psychology, 10, 403-420.

Eckhardt, C.I., Babcock, J. y Homack, S. (2004). Partner assaultive men and the stages and processes of change. Journal of Family Violence, 19, 81-93.

Escudero, A., Polo, C., López, M. y Aguilar, L. (2005). La persuasión coercitiva, modelo explicativo del mantenimiento de las mujeres en una situación de violencia de género. I: Las estrategias de la violencia. Revista de la Asociación Española de Neuropsiquiatría, 25, 85-120.

Expósito, F. y Ruiz, S. (2009). Reeducación de maltratadores: una experiencia de intervención desde la perspectiva de género. Intervención Psicosocial, 19, 145-151.

Feder, L. y Wilson, D.B. (2005). A meta-analytic review of court-mandated batterer intervention programs: Can courts affect abusers' behavior? Journal of Experimental Criminology, 1, 239-262.

Fernández-Montalvo, J., Echeburúa, E. y Amor, P.J. (2005). Aggressors against women in prison and in the community: an exploratory study of a differential profile. International Journal of Offender Therapy and Comparative Criminology, 49, 158-167.

Fernández-Montalvo, J., López-Goñi, J.J. y Arteaga, A. (2011). Tratamiento de agresores contra la pareja en programas de atención a drogodependientes: un reto de futuro. Adicciones, 23, 5-9.
Fernández-Montalvo, J., Echauri, J.A., Martínez, M. y Azcárate, J.M. (2011). Violencia de género e inmigración: perfil diferencial de hombres maltratadores nacionales e inmigrantes. Psicología Conductual, 19, 439-452.

Ferrer, V.A., Bosch, E., Navarro, C., Ramis, M.C. y García, M.E. (2008). Los micromachismos o microviolencias en la relación de pareja: Una aproximación empírica. Anales de Psicología, 24, 341-352.

Gondolf, E.W. (2004). Evaluating batterer counseling programs: A difficult task showing some effects and implication. Aggression and Violent Bebavior, 9, 605-631.

González de Rivera, J.L. (2002). Versión española del SCL-90-R. Madrid: TEA.

Hilton, N.Z, Harris, G.T y Rice, M.E. (2001). Predicting violence by serious wife assaulters. Journal of Interpersonal Violence, 16, 408-423.

Huss, M.T. y Ralston, A. (2008). Do batterer subtypes actually matter? Treatment completion, treatment response, and recidivism across a batterer typology. Criminal Justice and Behavior, 35, 710-724.

Lemire, G., Rondeau, G., Brochu, S., Schneeberger, P. y Brodeur, N. (1996). Les programmes de traitement pour hommes violents: du communautaire au correctionnel. Canadian Journal of Criminology, 38, 33-59.

Levesque, D.A., Velicer, W.F., Castle, P.H. y Greene, R.N. (2008). Resistance among domestic violence offenders: Measurement development and initial validation. Violence Against Women, 14, 158-184.

Lila, M., Herrero, J. y Gracia, E. (2008). Evaluating attribution of responsibility and minimization by male batterers: implications for batterer programs. The Open Criminology Journal, 1, 4-11.

Lila, M., Catalá, A., Conchell, R., García, A., Lorenzo, M.V., Pedrón, V. y Terreros, E. (2010). Una experiencia de investigación, formación e intervención con hombres penados por violencia contra la mujer en la Universidad de Valencia: Programa Contexto. Intervención Psicosocial, 19, 167-179.

Loinaz, I. (2010). El estudio de las tipologías de agresores de pareja en los centros penitenciarios. Ayudas a la investigación 2009. Barcelona: Centro de Estudios Jurídicos y Formación Especializada.

Loinaz, I., Echeburúa, E. y Torrubia, R. (2010). Tipología de agresores contra la pareja en prisión. Psicothema, 22, 106-111.

Loinaz, I., Irureta, M. y Domenech, F. (2011). Análisis de la reincidencia en agresores de pareja. Memoria de investigación 2010. Barcelona: Centro de Estudios Jurídicos y Formación Especializada.

Loinaz, I., Torrubia, R., Echeburúa, E., Navarro, J.C. y Fernández, L. (2009). Implicaciones de las tipologías de agresores de pareja para el tratamiento en prisión. Implications of intimate partner violence perpetrators' typologies for the treatment in prison. Cuadernos de Medicina Psicosomática $y$ Psiquiatría de Enlace, 91, 19-25.

Maiuro, R.D., Cahn, T.S. y Vitaliano, P.P. (1986). Assertiveness deficits and hostility in domestically violent men. Violence and Victims, 1, 279-289.

McConnaughy, E.A., DiClemente, C.C., Prochaska, J.O. y Velicer, W.F. (1989). Stages of change in psychotherapy: A follow-up report. Psychotherapy: Theory, Research and Practice, 26, 494-503.

Meis, L.A., Murphy, C.M. y Winters, J.J. (2010). Outcome expectancies of partner Abuse: assessing perpetrators' expectancies and their associations with readiness to change, abuse, and relevant problems. Assessment, 17, 30-43.

Montalvan, V., Graver, R., Oquendo, M.A., Baca-García, E., Morales, M. y Mann, J.J. (2001). Spanish adaptation of the Buss-Durkee Hostility Inventory (BDHI). European Journal of Psychiatry, 15, 101-112.

Montero, A. (2001). Síndrome de adaptación paradójica a la violencia doméstica: una propuesta teórica. Clínica y Salud, 12, 371-397.

Montero, I. y León, O.G. (2007). A guide for naming research studies in Psychology. International Journal of Clinical and Health Psychology, 7, 847862.

Murphy, C.M. y Meis, L.A. (2008). Individual treatment of intimate partner violence perpetrators. Violence and Victims, 23, 173-186.

Murphy, C.M. y Ting, L.A. (2010). Interventions for perpetrators of intimate partner violence: a review of efficacy research and recent trends. Partner Abuse, 1, 26-44.

Olson, D.E. y Stalans, L.J. (2001). Violent offenders on probation: Profile, sentence, and outcome differences among domestic violence and other violent probationers. Violence Against Women, 7, 1164-1185.

Olver, M.E., Stockdale, K.C. y Wormith, S. (2011). A meta-analysis of predictors of offender treatment attrition and its relationship to recidivism. Journal of Consulting and Clinical Psychology, 79, 6-21 
Pérez, M. y Martínez, M. (2010). La reincidencia de los condenados por delitos de violencia de género a programas formativos aplicados desde la ejecución penal en la comunidad. Barcelona: Centro de Estudios Jurídicos y Formación Especializada.

Pérez-Albéniz, A., De Paúl, J., Etxeberría, J., Montes, M.P. y Torres, E. (2003). Adaptación de Interpersonal Reactivity Index (IRI) al español. Psicothema, 15, 267-272.

Prochaska, J.O. y DiClemente, C.C. (1982). Transtheoretical therapy: Toward a more integrative model of change. Psychotherapy: Theory, Research and Practice, 19, 276-287.

Redondo, S. (2008). Manual para el tratamiento psicológico de los delincuentes. Madrid: Pirámide.

Redondo, N., Graña, J.L. y González, L. (2009). Características sociodemográficas y delictivas de maltratadores en tratamiento psicológico. Psicopatología Clínica, Legal y Forense, 9, 49-62.

Rojas, M.J. y Espinoza, L.S. (2008). Consejo psicológico y psicoterapia motivacional en drogodependencias: evaluación de las intervenciones del programa "Lugar de Escucha". Adicciones, 20, 185-196.

Romero, J., Rodríguez, M.J. y Echauri, J.A. (2005). Teoría y descripción de la violencia doméstica. Programa terapéutico para maltratadores del ámbi- to familiar en el centro penitenciario de Pamplona. Anuario de psicología jurídica, 15, 67-91.

Ross, E., Polaschek, D. y Ward, T. (2008). The therapeutic alliance: A theoretical revision for offender rehabilitation. Aggression and Violent Behavior 13, 462-480.

Sartin, R.M., Hansen, D.J. y Huss, M.T. (2006). Domestic violence treatment response and recidivism: A review and implications for the study of family violence. Journal of Aggression and Violent Behavior, 11, 425-440.

Saunders, D.G. (2008). Group interventions for men who batter: A summary of program descriptions and research. Violence and Victims, 23, 156-172.

Shamai, M. y Buchbinder E. (2010). Control of the self: partner-violent men's experience of therapy. Journal of Interpersonal Violence, 25, 13381362.

Taft, C.T., Murphy, C.M., Musser, P.H. y Remington, N.A. (2004). Personality, interpersonal, and motivational predictors of the working alliance in group cognitive-behavioral therapy for partner violent men. Journal of Consulting and Clinical Psychology, 72, 349-354.

(Artículo recibido: 24-05-2011, revisión: 18-01-2012, aceptado: 11-02-2012) 